\title{
Potential of Treating Age-Related Depression and Cognitive Decline with Nutraceutical Approaches: A Mini-Review
}

\author{
William O. Ogle Rachel B. Speisman Brandi K. Ormerod \\ J. Crayton Pruitt Family Department of Biomedical Engineering, Evelyn F. \& William L. McKnight Brain Institute, and \\ Institute on Aging, University of Florida, Gainesville, Fla., USA
}

\author{
Key Words \\ Polyphenolic phytochemicals · Curcumin · Resveratrol • \\ Depression - Stress $\cdot$ HPA axis $\cdot$ Serotonin $\cdot$ Adult \\ neurogenesis
}

\begin{abstract}
A variety of consumable plant-derived phytochemicals exhibit nutraceutical properties because they produce physiological benefits and combat disease processes. Emerging evidence suggests that widely accessible and safe organic polyphenolic phytochemicals, in particular, treat depression at much lower concentrations than clinical doses of classical drugs. Structurally similar polyphenolics such as curcumin, resveratrol, and proanthocyanidins exhibit antioxidant and immunomodulatory properties and recent research suggests that they also modulate hypothalamic-pituitary-adrenal (HPA) axis activity, serotonergic transmission and hippocampal neurogenesis (perhaps via their effects on serotonin and HPA activity). These data tempt speculation that polyphenolic compounds could also combat age-related cognitive decline, which is often accompanied by depression and potentially by reduced levels of hippocampal neurogenesis. Here we review the relationships between dysregulation of these systems and age-related cognitive decline. We then
\end{abstract}

\section{KARGER}

Fax +4161306 1234

E-Mail karger@karger.ch

www.karger.com
(C) 2012 S. Karger AG, Basel

0304-324X/13/0591-0023\$38.00/0

Accessible online at:

www.karger.com/ger suggest that this group of structurally similar polyphenolic compounds may be particularly promising therapeutic leads for age-related cognitive decline and depression because they modulate these processes.

Copyright $\odot 2012$ S. Karger AG, Basel

\section{Introduction}

Age-related cognitive decline is often accompanied and potentially exacerbated by depression, which can be mediated by dysregulated hypothalamic-pituitary-adrenal (HPA) axis and serotonergic activity, compromised neuronal plasticity and neurodegeneration $[1,2]$. These factors may independently or interdependently increase the burden of care for the aged individual as well as for their families and caregivers. Nutraceuticals are food products that are physiologically beneficial with defined effects on diseases [3]. Asian cultures have long recognized that polyphenolic compounds, such as curcumin, proanthocyanidins, ferulic acid and resveratrol and its metabolites piceatannol and pinostilbene (fig. 1) that protect plants from predation and pathogen invasion can effectively treat neuropsychiatric and neurodegenerative diseases. Evidence has accumulated over the past few de- 


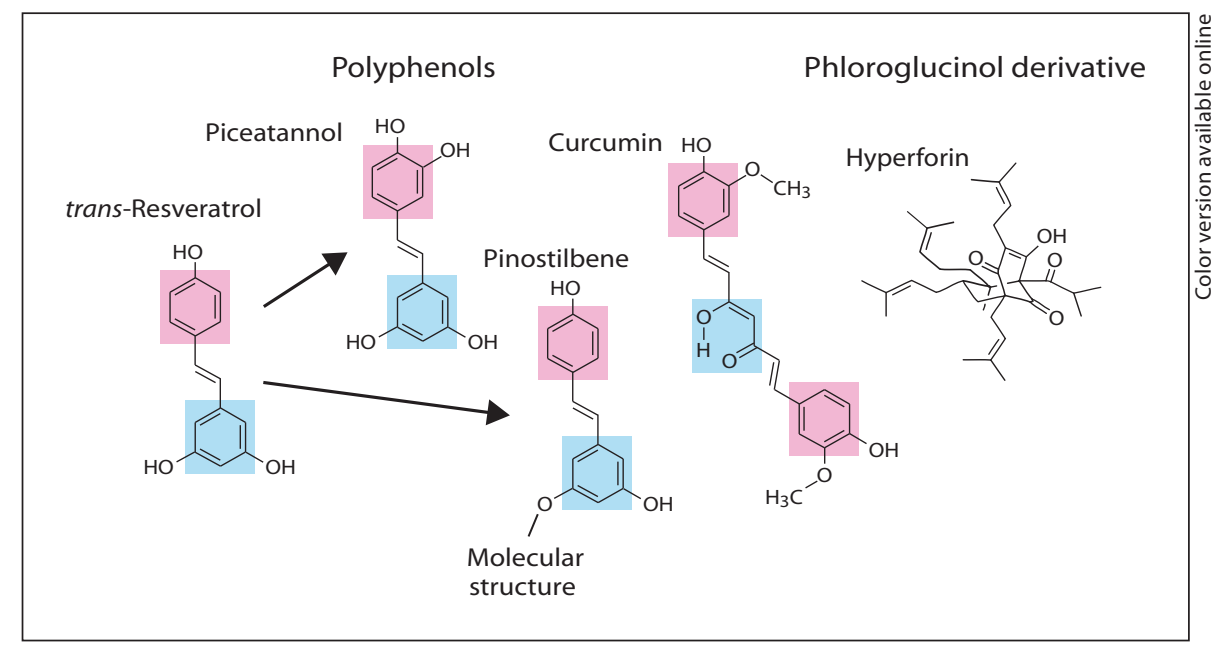

Fig. 1. Phenolic phytochemicals show promise as leads for treatments against depression. Emerging evidence shows that polyphenolic compounds similar structurally to curcumin mediate antidepressant effects in animal models by modulating 5 -HT receptor activity with fewer side effects than other molecules, such as hyperforin (the active ingredient in St. John's wort). Curcumin also stimulates forms of plasticity that include hippocampal neurogenesis and protects cells from the deleterious effects of stress

cades to suggest that these highly affordable and accessible polyphenolic phytochemicals may be safer alternatives to antidepressant drugs. In fact, they may alleviate depression by modulating HPA axis activity, neurotransmission and forms of plasticity that include hippocampal neurogenesis, which have all been strongly linked to depression [4].

Among members of the geriatric community institutionalized because of age-related cognitive decline $1-10 \%$ is estimated to suffer from major depression and up to $20 \%$ is estimated to suffer from a depressive disorder [5]. Disentangling whether depression precedes, correlates with or follows the physical, social and cognitive problems that often transpire with advanced age is difficult, but the strong link between depression and potentially reversible age-related cognitive decline (i.e. 'pseudo-dementia' [5]) suggests that effective treatments for both problems [4] that are accessible to our rapidly growing elderly population are critical. Polyphenolic phytochemicals may be particularly attractive for preventing and possibly treating geriatric depression because they modulate HPA axis hyperactivity, while stimulating neurotransmission and forms of neural plasticity that include hippocampal neurogenesis, which have also been hypothesized to underlie age-related cognitive decline $[1,2,4-6]$. potentially by stimulating neurotrophin release. Although the effects of other polyphenolic compounds that include resveratrol (and its metabolites pinostilbene and piceatannol) on depression, HPA axis hyperactivity, neurotrophin levels, and cell death are only beginning to be investigated, emerging functional similarities suggest that their structural similarities (highlighted in pink and blue; colors refer to online version only) may underlie their effects.

\section{Links between Stress, Depression and Cognition across the Lifespan}

\section{Serotonergic Neurotransmission, Depression and} Cognition across the Lifespan

Dysregulated serotonergic transmission has been long implicated in depression. Serotonergic raphe neurons project to diverse brain regions that control a wide range of physiological and cognitive processes that are disrupted in depression. Serotonin (5-HT) interacts with receptors that include the ionotropic $5-\mathrm{HT}_{3}$ receptor and metabotropic $G$ protein-coupled receptors (5- $\mathrm{HT}_{1 \mathrm{~A}-\mathrm{F}, \mathrm{P}, \mathrm{S}}, 5-\mathrm{HT}_{2 \mathrm{~A}-\mathrm{C}}, 5-\mathrm{HT}_{4}, 5-\mathrm{HT}_{5}, 5-\mathrm{HT}_{6}, 5-\mathrm{HT}_{7}$ ) that activate intracellular second messenger cascades [7]. The $5-\mathrm{HT}_{1 \mathrm{~A}}$ and $5-\mathrm{HT}_{2 \mathrm{~A}}$ receptors (and potentially $5-\mathrm{HT}_{1 \mathrm{~B}}$ and $5-\mathrm{HT}_{2 \mathrm{C}}$ receptors with affinities for their respective ligands) are most often implicated in depressive disorders because they are expressed in brain regions that exhibit morphological and biochemical changes in depressed patients and because anti-depressant drugs prolong their interaction with $5 \mathrm{HT}$ either directly or by inactivating nearby 5 -HT transporters. Moreover, 5- $\mathrm{HT}_{1 \mathrm{~A}}$ autoreceptor expression may be enriched on serotonergic midbrain raphe neurons that project to the prefrontal cortex and hippocampus that 
can exhibit altered $5 \mathrm{HT}_{1 \mathrm{~A}}$ and $5 \mathrm{HT}_{/ 2 \mathrm{~A}}$ postsynaptic receptor densities $[2,4,7]$.

Current antidepressant strategies are more selective refinements of the original tricyclic and monoamine oxidase inhibitor (MAOI) antidepressant strategies that prolonged synaptic monoamine activity. For example, fewer side effects are associated with selective serotonin reuptake inhibitors (SSRIs), noradrenaline reuptake inhibitors (NRIs) and serotonin and noradrenaline reuptake inhibitors (SNRIs) than with traditional anti-depressants. Full or partial 5- $\mathrm{HT}_{1 \mathrm{~A}}$ receptor antagonism is sometimes used to improve the speed and efficacy of reuptake strategies and $5-\mathrm{HT}_{2 \mathrm{~A} / 2 \mathrm{C}}$ receptor antagonism has been employed to minimize the side effects that persist even with SSRI and SNRI medications. The need for new strategies is apparent in the observation that even after several weeks of treatment with and without combined psychotherapy, these drugs are only fully effective in about $50 \%$ of patients [7]. Data showing that these antidepressants can reverse the prefrontal cortical and hippocampal atrophy observed in depressed patients are interesting because they suggest that dysregulated serotonergic transmission interacts with HPA axis hyperactivity, which is thought to the mediate the hippocampal atrophy [2].

Somewhat controversial evidence suggests that serotonergic neurotransmission changes with age. A few postmortem examinations have detected decreased densities and affinities among $5-\mathrm{HT}_{1 \mathrm{~A}}, 5-\mathrm{HT}_{1 \mathrm{~B} / \mathrm{D}}$, and $5-\mathrm{HT}_{2 \mathrm{~A}}$ receptor proteins expressed in cortical, occipital and hippocampal regions across life in humans, and particularly in women. These age-related disruptions in serotonergic neurotransmission have been hypothesized to predispose the elderly to depression and appear to correlate with changes in cholinergic activity, which has been implicated in age-related cognitive decline. Although the role that the hippocampus plays in depression is unclear, age-related declines in hippocampal 5HT receptors densities are interesting because the hippocampus mediates forms of learning and memory that are also compromised by age $[1,11,13]$.

\section{HPA Axis Hyperactivity, Depression and Age-Related Cognitive Decline}

HPA axis dysregulation has been linked to both depression and age-related cognitive decline [2]. Everyday environmental or psychological stimuli that activate the HPA axis, the autonomic nervous system and the immune system are called stressors. HPA axis activation in the face of a stressor promotes survival by mobilizing energy and increasing cardiovascular tone while suppress- ing responses that do not promote survival (i.e. tissue repair). Glucocorticoids, such as corticosterone, diffuse passively into the brain and stress-level glucocorticoids readily occupy and activate glucocorticoid receptor proteins (GRs) expressed in limbic regions such as the hippocampus, hypothalamus, locus coeruleus and the dorsal raphe nucleus. Corticosterone-bound GRs translocate into cell nuclei as transcription factors that alter the expression of genes controlling processes that include neuronal excitability, neurochemistry and structural plasticity. Normally, corticosterone-bound hypothalamic and pituitary GRs respectively shunt the corticotropin-releasing hormone $(\mathrm{CRH})$ and then adrenocorticotrophic hormone $(\mathrm{ACTH})$ production that stimulates adrenal corticosterone secretion, rendering stress-increased corticosterone levels short-lived [2].

When the glucocorticoid mediators of stress-activated systems are not restored to homeostatic levels either upon removal of the stressor or because of steady streams of actual or perceived stressors, the effects may become deleterious [2]. Chronically elevated corticosterone levels are hypothesized to eventually impair HPA axis feedback inhibition by desensitizing the GRs that they interact with in the CNS, including the hippocampus. Indeed, corticosterone levels in rats and cortisol levels in primates that have been elevated chronically by repeated exposures to stressors are a hallmark of impaired HPA axis feedback inhibition. The long-term consequences of these repeated exposures include compromised synaptic transmission, impaired epithelial cell growth, reduced energy metabolism and hippocampal atrophy associated with dendritic pruning and cell death.

Depression is often associated with the inability to cope with streams of stressors, potentially because chronic stress-potentiated glucocorticoid levels compromise central nervous system (CNS) integrity $[2,4,16]$. Indeed, HPA axis hyperactivity is reported in the majority of depressed patients. Glucocorticoid hyper-secretion is associated with impaired memory and reduced hippocampal volumes in aged rats and humans. For example, reducing glucocorticoid levels via adrenalectomy in middle age preserves memory and hippocampal integrity through life, while elevating glucocorticoids with chronic stress paradigms accelerates the expression of aging biomarkers in rats. Furthermore, significant age-related increases in diurnal cortisol elevations are associated with smaller hippocampal volumes and lower memory test scores in humans. Therefore, therapeutics targeting HPA axis hyperactivity may be extremely attractive for treating agerelated cognitive decline and depression because age-re- 
lated HPA axis hyperactivity is associated with depression, hippocampal atrophy and impaired cognition.

\section{Overlap between HPA Axis Activity and Serotonergic} Transmission: Implications for Aging

Although the effects of dysregulated serotonergic transmission and HPA axis hyperactivity on depression are typically investigated independently, several lines of evidence suggest that their effects overlap $[8,9]$. First, serotonin influences diverse behaviors such as mood, sleep and sexual behavior that are also modulated by HPA axis activation. Second, similar structural alterations are observed in models of stress-induced depression, chronic HPA axis hyperactivity and depression. For example, chronic HPA axis activation following repeated exposures to inescapable stressors increases 5-HT and 5-HT receptor turnover in the frontal cortex, hippocampus and amygdala, among other rat brain regions. In the rat, adrenalectomy is followed by a rapid and marked increase in limbic $5-\mathrm{HT}_{1 \mathrm{~A}}$ receptor transcription that is completely reversed by corticosterone activity at both the mineralocorticoid receptor and GR. Glucocorticoid-inducing stress exposure also decreases the affinity of $5-\mathrm{HT}_{1 \mathrm{~A}}$ receptors for ligand. On the other hand, $5-\mathrm{HT}_{1 \mathrm{~A}}$, hypothalamic $5-\mathrm{HT}_{2 \mathrm{C}}$ or $5-\mathrm{HT}_{7}$ (and possibly $5-\mathrm{HT}_{2 \mathrm{~A}}$ and $5-\mathrm{HT}_{1 \mathrm{~B}}$ ) agonists increase $\mathrm{ACTH}, \mathrm{CRH}$ and plasma corticosterone or cortisol levels in animals and in humans, respectively. Interestingly, serotonergic neurotransmission and HPA axis activity modulate forms of plasticity that include adult hippocampal neurogenesis, which has been linked both to depression and more recently to age-related cognitive decline [6, 10-12]. Although the idea that age-related changes in serotonergic transmission could accompany age-related HPA axis hyperactivity is highly speculative, it could explain the elevated incidence of depressive disorders among cognitively impaired elderly patients.

\section{Neurotrophic Hypothesis of Depression and Adult Neurogenesis}

The neurotrophic hypothesis of depression was formulated by several lines of research. Small molecule neurotrophins regulate the differentiation, survival and synaptic and morphological plasticity of neurons. The hippocampal and frontal cortical atrophy observed in depressed patients and in animal models of depression suggests that neuronal survival and structural plasticity may be compromised. Indeed, postmortem examinations have revealed reduced hippocampal brain-derived neurotrophic factor (BDNF) levels in some depressed pa- tients unless they were on an antidepressant regimen just before death $[4,14]$. In laboratory animals, the deleterious effects of chronic stress on behavior and BDNF levels can be reversed by antidepressants. Midbrain or hippocampal BDNF infusion produces antidepressant-like effects in behavioral tests of depression unless the animals are mutant for either BDNF or its cognate tyrosine kinase B (TrkB) receptor protein. Moreover, exposure to stress or high-level glucocorticoids not only decreases BDNF levels but also levels of nerve growth factor, neurotrophin-3 and fibroblast growth factor. These factors regulate several forms of neural plasticity that includes hippocampal neurogenesis, which has been linked to both depression and age-related cognitive decline $[6,10,12]$.

New neurons are added each day through life to the hippocampal dentate gyri of all mammals studied, including humans [10]. The effect of new neurons on hippocampal integrity remains unclear, but rates of neurogenesis detected in the dentate gyri of young rodents, dogs and nonhuman primates generally correlate with behavioral measures in hippocampus-dependent tasks [11]. In young rodents, exposure to repeated stressors or high-level glucocorticoids decreases hippocampal neurogenesis [10], which is interesting because HPA axis hyperactivity appears to be a natural consequence for aging humans [2] and decreased rates of neurogenesis in the aging rodent brain can be reversed by adrenalectomy with low-level corticosterone replacement [6]. Measures of hippocampal neurogenesis and performance in tasks that require the rapid acquisition of spatial information appear linked in aged rats $[11,12]$, suggesting that preserving neurogenesis through life may also preserve some forms of cognition. In fact, neurons produced in the young and aged hippocampus resemble one another functionally [10] and exposure to an enriched environment can reverse age-related impairments in both neurogenesis and cognition [11].

The role that the hippocampus plays in depression is unclear, but the hippocampal atrophy observed in patients with clinical depression or post-traumatic stress disorder and in animal models of stress-induced depression suggests that reduced hippocampal integrity precedes, accompanies or follows depression [15-17]. 5- $\mathrm{HT}_{1 \mathrm{~A}}$ receptors that are typically expressed robustly in the hippocampus, including the dentate gyrus where neurogenesis occurs, are down-regulated in animal models of depression. Treatment with a variety of anti-depressants that include tricyclics, SSRIs, NRIs, MAOIs, electroconvulsive shock and lithium for durations sufficient to produce clinical effects (2-4 weeks) upregulate hippocampal 
neurogenesis most likely through $5-\mathrm{HT}_{1 \mathrm{~A}}$ receptor signaling in corticosterone-treated, chronically stressed and naïve animals. While ablating neurogenesis with targeted $\gamma$-irradiation or genetic mutation does not appear to produce depressive behaviors in rodents, the behavioral effects of anti-depressants appear to require the production of new hippocampal neurons. Indeed, neural progenitor cell proliferation is decreased by $5-\mathrm{HT}_{1 \mathrm{~A}}$ receptor antagonists, lesions of hippocampal 5-HT input and in mice mutant for $5-\mathrm{HT}_{1 \mathrm{~A}}$ receptor proteins. Thus, age-related changes in serotonergic transmission and hippocampal neurogenesis likely impact the onset and progression of depression, forms of cognition that rely upon hippocampal integrity as well as decrease antidepressant efficacy.

Chronic treatment with antidepressants that modulate 5-HT receptor activity increases levels of hippocampal BDNF, cAMP-responsive element-binding protein (CREB) mRNA, protein and phosphorylation. In accordance, BDNF and CREB levels are decreased in cerebral cortex of depressive patients and antidepressant treatment can enhance BDNF levels in depressive patients. Once dimerized with TrkB, BDNF produces diverse physiological effects through Ras-Raf-MAPK (aka ERK), $\mathrm{IP}^{3} \mathrm{~K}-\mathrm{AKT}$ and PLC $\gamma$ signaling pathways that include CREB activation. The activation of hippocampal CREB signaling has been shown to potentiate hippocampal neurogenesis, produce antidepressant effects in the forced swim test and to induce BDNF expression, which also stimulates hippocampal neurogenesis and produces antidepressant effects in animal models of depression. In CREB knockout mice, BDNF upregulation is abolished after antidepressant treatment. Reduced BDNF production under acute or chronic stress situations can reduce rates of neurogenesis but increase neuronal pruning and both phenomena can be reversed with antidepressants [15-17]. Clearly, the effects of depression on the expression of neurotrophic factors and neurogenesis could precede or even exacerbate age-related cognitive decline. Therapeutic strategies capable of stimulating neurogenesis may, therefore, alleviate both depressive symptoms and cognitive impairment in elderly patients.

\section{Could Polyphenolic Nutraceuticals Combat Depression and Age-Related Cognitive Decline?}

\section{Effects of Polyphenolic Phytochemicals on Cognition and Depression}

The health benefits of a diet enriched in unprocessed plants are likely mediated by the degree of diversity among bioactive phytochemical (or nutraceutical) compounds they contain (for excellent comprehensive reviews, see $[3,18])$. In fact, the risk of chronic diseases, including coronary heart disease and some cancers, correlates negatively with the diversity of plants consumed by low-risk populations. These data suggest the presence of relationships between food diversity, chemical diversity among these foods and the subsequent effects of those compounds on biological activity within members of these low-risk populations. Plants have evolved defensive and adaptive chemical mechanisms to stave off predation and the encroachment of diverse competitive species. Importantly, organisms that consume these plants despite their antipredatory mechanisms have likely adapted counteractive mechanisms. A major question is whether these phytochemicals or the adaptive mechanisms they may stimulate upon consumption provide a health benefit to the organism. Indeed, phenylpropanoids that include polyphenols (stilbenes and anthocyanins) and tannins (proanthocyanidins) and which are enriched in foods such as grapes and red wines, as well as dietary indoleamines such as melatonin and serotonin found in a variety of plants that include grapes, are often implicated in diets thought to promote health.

The three most common classes of phytochemical found in medicinal herbs are the phenylpropanoids, isoprenoids, and alkaloids. The related compounds curcumin, resveratrol, fisetin and proanthocyanidins are phenylpropanoids composed of more than one phenolic ring (polyphenols; fig. 1) that are quite often identified as the active ingredients in medicinal plants with the ability to modulate the activity of a variety of enzymes and receptor proteins. They are specifically categorized by the number of phenol rings and the structural elements linking the phenolic rings, but are interestingly all structurally similar derivatives of hydroxycinnamic acids, cinnamic aldehydes, monolignols, coumarins, flavonoids and stilbenoids.

Some phytochemicals have shown efficacy for treating neuropsychiatric diseases in clinical trials. For example, a meta-analysis of 29 trials demonstrated that St. John's wort (Hypericum perforatum L.) is not only superior to placebo and as effective as tricyclic and SSRI antidepressants for treating depression but produces side effects in significantly fewer patients than standard antidepressants [19]. However, St. Johns wort and its active ingredient hyperforin may interact with many of the medications often prescribed to elderly patients and its antagonistic effects on choline reuptake may aggravate age-related cognitive decline [20]. Interestingly, the con- 
sumption of a mixture of choline, uridine, and docosahexaenoic acid (DHA), along with B vitamins appears to improve memory in mild and very mild cases of Alzheimer's disease, potentially by stimulating cellular and synaptic plasticity [21]. The effects of this mixture on cellular and synaptic plasticity suggest that it may also be beneficial for age-related cognitive decline. Olive oil and its putative active ingredient hydroxytyrosol exhibit neuroprotective properties both in vivo and in vitro and epidemiological studies suggest that diets rich in olive oil slow the onset and progression of age-related cognitive decline and Alzheimer's disease [18]. In animal studies, a handful of structurally similar readily consumable polyphenols that include curcumin, resveratrol and its pinostilbene and piceatannol metabolites, proanthrocyanidin and ferulic acid are emerging as therapeutic targets for depression. Because they appear to modulate serotonergic transmission, HPA axis activity and neurogenesis, which may all be implicated in age-related cognitive decline, these polyphenols may be particularly attractive for treating geriatric depression because they may also combat age-related cognitive decline through their effects on these systems [10-12].

Curcumin (a.k.a. turmeric or Tuber curcumae) is the major active ingredient of Curcuma longa, a perennial herb of the ginger family. The health benefits of curcumin have been explored far more extensively than those of other emerging polyphenolics. Curcumin has been a staple of traditional Indian, Indonesian and Chinese medicines for thousands of years. Yu Jin, the Chinese version of curcumin, is used routinely to treat depression, depressive disorders and insomnia. Doses of curcumin thought to inhibit monoamine oxidase levels reduce behaviors associated with anxiety/depression in both the forced swimming test and in a chronic unpredictable stress model [17]. The antidepressant effects of subclinical doses of some anti-depressant drugs (i.e. fluoxetine, venlafaxine and buproprion but not desipramine) are potentiated by curcumin, suggesting that it modulates serotonergic, dopaminergic and perhaps noradrenergic transmission. The finding that prefrontal cortical serotonin and dopamine levels and prefrontal cortical/striatal levels of noradrenaline increase in curcumin-treated mice supports this notion. Interestingly, the effects of curcumin on behavior in tasks measuring anxiety/depression and on cortical serotonin, dopamine and noradrenaline levels can be potentiated by the bioavailability enhancer piperine [22]. Pharmacological studies suggest that the major antidepressant effects of curcumin are mediated through serotonergic transmission, most likely at
5- $\mathrm{HT}_{1 \mathrm{~A} / 1 \mathrm{~B}}$ and 5- $\mathrm{HT}_{2 \mathrm{C}}$ subtypes. Indeed, curcumin can even attenuate stress-induced decreases in hippocampal 5- $\mathrm{HT}_{1 \mathrm{~A}}$ mRNA levels [23].

Another extensively studied polyphenolic phytochemical is the stilbenoid resveratrol found enriched in the seeds and skin of fruits, such as grapes, and at lower levels in red wine. trans-Resveratrol, isolated from the Japanese knotweed tree (Polygonum cuspidatum), is a traditional Chinese medicine known as $\mathrm{Hu}$ Zhang that is commonly used to treat inflammation, viral infections, cancer and neuropsychiatric disorders among other ailments. In animal experiments, resveratrol has been shown to reduce inflammation and protect neurons from death in in vitro and in vivo models of Alzheimer's disease and ischemia. Resveratrol may also preserve learning and memory from the effects of lesions by minimizing cell death and oxidative stress among neurons surviving the lesion. Recent studies have also shown that resveratrol can inhibit noradrenaline and 5-HT reuptake in rats and significantly decrease anxiety/depressive behaviors while increasing hippocampal serotonin and noradrenaline levels in mice [24]. The decrease in brain monoamine oxidase suggests that the dietary intake of resveratrol can increase 5-HT activity within the brain supporting the notion that resveratrol may possess potent antidepressant properties.

Finally, proanthocyanidins or oligomeric proanthocyanidins are condensed tannins (oligomer chains of flavonols) found in many plants including blueberries, grape seeds and skin sas well as red wine. Proanthocyanidins extracted from grape seeds, the bark of the Chinese Scutellaria baicalensis herb or the Brazilian Croton celtidifolius Baill tree exert potent anti-inflammatory, antioxidant, antinociceptive and cardioprotective/vasodilation effects and may exhibit antidepressant properties. For example, the duration of immobility in both tail suspension and forced swimming tests of anxiety/depressive behavior was reduced in mice treated with grape seed extract (with $\sim 90 \%$ proanthocyanidin and $\sim 7 \%$ flavonoid content) versus vehicle that also have elevated 5-HT levels in their frontal cortices, hippocampi and hypothalami [25]. Taken together, these data suggest that related polyphenolic compounds may exhibit anti-depressant properties, along with other properties that may reverse age-related cognitive decline. For example, curcumin's positive effect on neurogenesis is interesting because this drastic form of neuroplasticity is downregulated in models of depression and across age. Whether curcumin's antidepressant action is, at least in part, mediated by its effects on neurogenesis is unclear, but the link between neurogenesis 


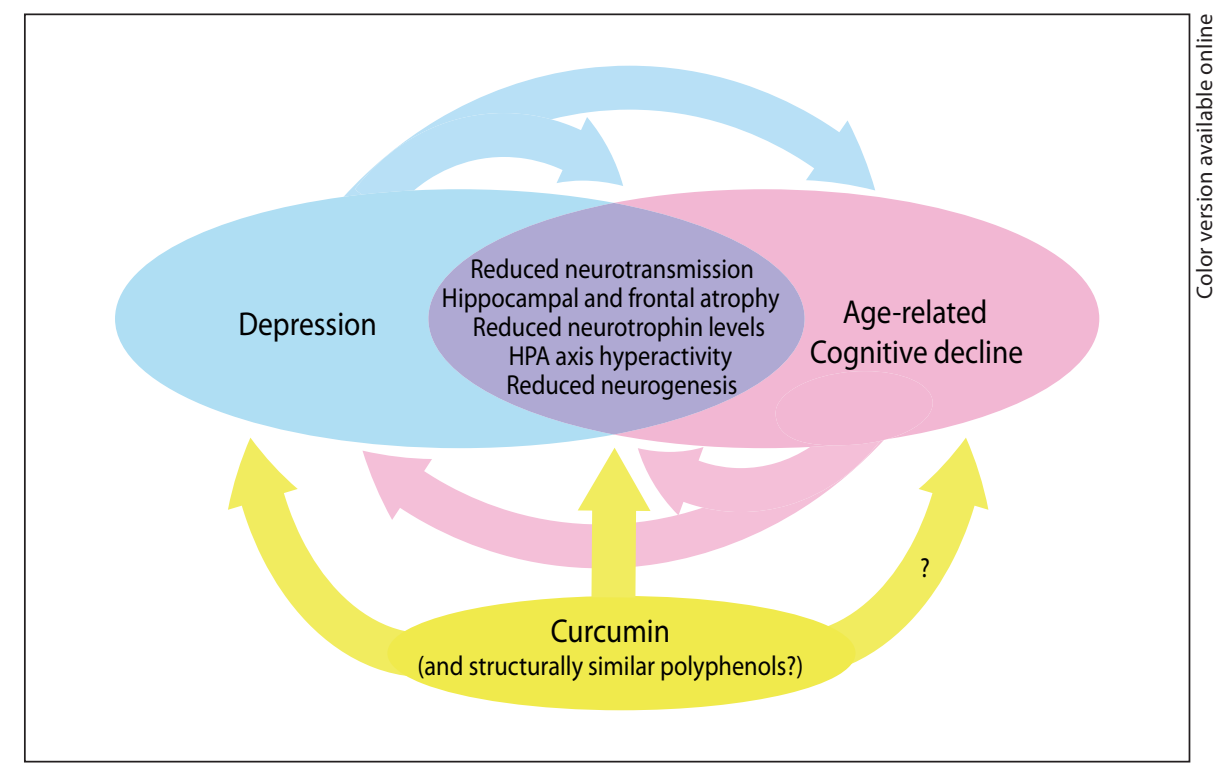

Fig. 2. The effects of polyphenolics such as curcumin on depression and potentially age-related cognitive decline could be linked through a number of factors that include HPA axis hyperactivity and compromised neurotransmission, neuronal plasticity and neuronal survival. Curcumin appears to prevent chronic stressinduced depressive behaviors and stress-induced compromises in neurotransmission, neuronal plasticity (including hippocampal neurogenesis) and cell death in several culture models. These phenomena have been shown to occur in both animal models of depression and age-related cognitive decline, suggesting that curcumin and structurally related molecules may be extremely beneficial for geriatric depression because they may also treat agerelated cognitive decline. and hippocampal function in young and aged rodents suggests that its effects on this drastic form of neural plasticity may be beneficial for cognition.

\section{Neurotrophic and Neuroprotective Properties of \\ Phytochemicals}

A growing body of research has highlighted the neuroprotective and neurotrophin-stimulating properties of polyphenolic compounds that we suggest may also exhibit antidepressant properties. These compounds may be particularly attractive leads for geriatric depression because they may also treat the compromised plasticity and atrophy that may contribute to age-related cognitive decline [1]. For example, curcumin potentiates hippocampal neurogenesis and protects both BDNF levels and hippocampal neurogenesis from the deleterious effects of chronic stress [23]. Curcumin-stimulated BDNF signaling may also minimize glutamate-induced excitotoxic cell death among cortical primary cells [26]. Resveratrol can restore hippocampal volumes and hippocampal neurogenesis while minimizing cell death in mouse models of chronic fatigue syndrome and cerebral ischemia $[27,28]$. Some recent research even suggests that the resveratrol metabolites pinostilbene $\left(3,4^{\prime}\right.$-dihydroxy5-methoxy-trans-stilbene) and piceatannol (3,4,3',5'tetrahydroxy-trans-stilbene) are neuroprotective because they protect SH-SY5Y cells from 6-hydroxydopamine-induced neurotoxic cell death and PC-12 cells from $A \beta$-amyloid-induced neuronal cell death, respectively [29]. While the mechanisms mediating the beneficial effects of curcumin, resveratrol and the metabolites of resveratrol are under investigation, their known pharmacological properties strongly suggest their promise as novel leads for the treatment of neurological decline as well as depression.

Additional phytochemicals are also emerging as novel leads for treatments against neurological decline. The flavonol fisetin $\left(3,3^{\prime}, 4^{\prime} 7^{\prime}\right.$-tetrahydroxylflavone) abundant in many plants that include acacia, strawberries and mangoes protects neuronal cells from oxidative stressinduced cell death and can promote neuronal differentiation among cultured cells by activating MAPK signaling pathways. Fisetin has also been shown to promote longterm potentiation and memory as well as to reduce postischemia infarct size in rats [30]. Ferulic acid (4-hydroxy3 -methoxycinnamic acid) is abundant in vegetables and 
exhibits strong structural similarities to fisetin, resveratrol and curcumin, but is composed of a single phenolic group. Ferulic acid exhibits antioxidative, anti-inflammatory, anticancer, antidiabetic, anti-atherogenic and neuroprotective effects [31]. In addition, ferulic acid protects neurons from $A \beta$-amyloid induced cell death and attenuates stress-induced decreases in BDNF mRNA levels and depressive behavior in mice. Future work testing whether fisetin and ferulic acid, as well as other phytochemicals that structurally resemble curcumin and resveratrol, exhibit similar neuroprotective properties and can stimulate plasticity would be an interesting assessment of their relevance as leads for treating depression as well as neurologic decline across lifespan.

\section{Future Directions: Treating Age-Related Cognitive Decline and Depression with Polyphenolics}

Evidence demonstrating the anti-oxidant and anti-inflammatory properties of polyphenolic phytochemicals has been widely accepted. More recent evidence suggests that polyphenolic phytochemicals that exhibit structural similarities to curcumin exhibit anti-depressant properties that may be linked to their effects on serotonergic neurotransmission, HPA axis activity and forms of plasticity that include hippocampal neurogenesis. In fact, these polyphenolic phytochemicals may be particularly attractive therapeutic targets for treating geriatric depression because HPA axis hyperactivity, compromised neuronal plasticity and dysregulated monoaminergic neurotransmission have also been linked to age-related cognitive decline (fig. 2). Whether depression precedes, correlates with or follows age-related cognitive decline is unclear but the effects of polyphenolic compounds on correlates of depression and age-related cognitive decline are encouraging. Theoretically, phytochemical-based treatments for geriatric depression and cognitive decline could be moved rapidly to clinical trials on humans because of their high bioavailability and low toxicity, which are particularly beneficial features for pharmacotherapies intended for geriatric populations. Future studies addressing whether short-term or long-term dietary intake of these compounds as natural food sources or as supplements can reduce the incidence or severity of depression and/or age-related cognitive decline appear critical.

\section{Acknowledgements}

This work was supported by grants from the Ellison Medical Foundation to W.O.O. and by the Broad Foundation for Biomedical Research and the Evelyn F. McKnight Brain Research Foundation to B.K.O. and an N.S.F. graduate fellowship to R.B.S.

\section{References}

1 Foster TC: Biological markers of age-related memory deficits: Treatment of senescent physiology. CNS Drugs 2006;20:153-166.

-2 Lupien SJ, McEwen BS, Gunnar MR, Heim C: Effects of stress throughout the lifespan on the brain, behaviour and cognition. Nat Rev Neurosci 2009;10:434-445.

-3 Calabrese V, Cornelius C, Mancuso C, Pennisi G, Calafato S, Bellia F, Bates TE, Giuffrida Stella AM, Schapira T, Dinkova Kostova AT, Rizzarelli E: Cellular stress response: a novel target for chemoprevention and nutritional neuroprotection in aging, neurodegenerative disorders and longevity. Neurochem Res 2008;33:2444-2471.

4 Berton O, Nestler EJ: New approaches to antidepressant drug discovery: beyond monoamines. Nat Rev Neurosci 2006;7:137-151.

5 Meltzer CC, Smith G, DeKosky ST, Pollock BG, Mathis CA, Moore RY, Kupfer DJ, Reynolds CF: Serotonin in aging, late-life depression, and Alzheimer's disease: the emerging role of functional imaging. Neuropsychopharmacology 1998;18:407-430.
-6 Lazarov O, Mattson MP, Peterson DA, Pimplikar SW, van Praag H: When neurogenesis encounters aging and disease. Trends Neurosci 2010;33:569-579.

7 Carr GV, Lucki I: The role of serotonin receptor subtypes in treating depression: a review of animal studies. Psychopharmacology (Berl) 2011;213:265-287.

-8 Lanfumey L, Mongeau R, Cohen-Salmon C, Hamon M: Corticosteroid-serotonin interactions in the neurobiological mechanisms of stress-related disorders. Neurosci Biobehav Rev 2008;32:1174-1184.

\9 Xu Y, Zhang C, Wang R, Govindarajan SS, Barish PA, Vernon MM, Fu C, Acharya AP, Chen L, Boykin E, Yu J, Pan J, O’Donnell JM, Ogle WO: Corticosterone induced morphological changes of hippocampal and amygdaloid cell lines are dependent on 5-HT7 receptor related signal pathway. Neuroscience 2011;182:71-81.

10 Deng W, Aimone JB, Gage FH: New neurons and new memories. How does adult hippocampal neurogenesis affect learning and memory? Nat Rev Neurosci 2010;11:339-350.
11 Speisman R, Kumar A, Rani A, Foster TC, Ormerod BK: Environmental enrichment restores neurogenesis and rapid acquisition in aged rats. Neurobiol Aging 2012 Jul 11 [Epub ahead of print].

12 Dupret D, Revest JM, Koehl M, Ichas F, De Giorgi F, Costet P, Abrous DN, Piazza PV: Spatial relational memory requires hippocampal adult neurogenesis. PLoS One 2008; 3:e1959.

13 Eisch AJ, Cameron HA, Encinas JM, Meltzer LA, Ming GL, Overstreet-Wadiche LS: Adult neurogenesis, mental health, and mental illness. Hope or hype? J Neurosci 2008;28: 11785-11791.

14 Voleti B, Duman RS: The roles of neurotrophic factor and wnt signaling in depression. Clin Pharmacol Ther 2012;91:333-338.

15 David DJ, Wang J, Samuels BA, Rainer Q, David I, Gardier AM, Hen R: Implications of the functional integration of adult-born hippocampal neurons in anxiety-depression disorders. Neuroscientist 2010;16: 578-591. 
16 Schmidt HD, Shelton RC, Duman RS: Functional biomarkers of depression: diagnosis, treatment, and pathophysiology. Neuropsychopharmacology 2011;36:2375-2394.

-17 Xu Y, Barish PA, Pan J, Ogle WO, O’Donnell JM: Animal models of depression and neuroplasticity: assessing drug action in relation to behavior and neurogenesis. Methods $\mathrm{Mol}$ Biol 2012;829:103-124.

18 Schaffer S, Asseburg H, Kuntz S, Muller WE, Eckert GP: Effects of polyphenols on brain ageing and Alzheimer's disease: focus on mitochondria. Mol Neurobiol 2012, E-pub ahead of print.

19 Linde K, Berner MM, Kriston L: St John's wort for major depression. Cochrane Database Syst Rev 2008:CD000448.

20 Izzo AA, Ernst E: Interactions between herbal medicines and prescribed drugs: an updated systematic review. Drugs 2009;69:17771798.

-21 Wurtman RJ, Cansev M, Sakamoto T, Ulus I: Nutritional modifiers of aging brain function: use of uridine and other phosphatide precursors to increase formation of brain synapses. Nutr Rev 2010;68(suppl 2):S88S101.
22 Bhutani MK, Bishnoi M, Kulkarni SK: Antidepressant like effect of curcumin and its combination with piperine in unpredictable chronic stress-induced behavioral, biochemical and neurochemical changes. Pharmacol Biochem Behav 2009;92:39-43.

$23 \mathrm{Xu} \mathrm{Y,} \mathrm{Ku} \mathrm{B,} \mathrm{Cui} \mathrm{L,} \mathrm{Li} \mathrm{X,} \mathrm{Barish} \mathrm{PA,} \mathrm{Foster} \mathrm{TC,}$ Ogle WO: Curcumin reverses impaired hippocampal neurogenesis and increases serotonin receptor 1a mRNA and brain-derived neurotrophic factor expression in chronically stressed rats. Brain Res 2007;1162:9-18.

24 Xu Y, Wang Z, You W, Zhang X, Li S, Barish PA, Vernon MM, Du X, Li G, Pan J, Ogle WO: Antidepressant-like effect of trans-resveratrol: involvement of serotonin and noradrenaline system. Eur Neuropsychopharmacol 2010;20:405-413.

25 Xu Y, Li S, Chen R, Li G, Barish PA, You W, Chen L, Lin M, Ku B, Pan J, Ogle WO: Antidepressant-like effect of low molecular proanthocyanidin in mice: involvement of monoaminergic system. Pharmacol Biochem Behav 2010;94:447-453.

26 Wang R, Li YH, Xu Y, Li YB, Wu HL, Guo H, Zhang JZ, Zhang JJ, Pan XY, Li XJ: Curcumin produces neuroprotective effects via activating brain-derived neurotrophic factor/TrkB-dependent MAPK and PI-3K cascades in rodent cortical neurons. Prog Neuropsychopharmacol Biol Psychiatry 2010;34: 147-153.
27 Moriya J, Chen R, Yamakawa J, Sasaki K, Ishigaki Y, Takahashi T: Resveratrol improves hippocampal atrophy in chronic fatigue mice by enhancing neurogenesis and inhibiting apoptosis of granular cells. Biol Pharm Bull 2011;34:354-359.

28 Della-Morte D, Dave KR, DeFazio RA, Bao YC, Raval AP, Perez-Pinzon MA: Resveratrol pretreatment protects rat brain from cerebral ischemic damage via a sirtuin 1-uncoupling protein 2 pathway. Neuroscience 2009;159:993-1002.

29 Chao J, Li H, Cheng KW, Yu MS, Chang RC, Wang M: Protective effects of pinostilbene, a resveratrol methylated derivative, against 6-hydroxydopamine-induced neurotoxicity in SH-SY5Y cells. J Nutr Biochem 2010;21: 482-489.

30 Maher P: A comparison of the neurotrophic activities of the flavonoid fisetin and some of its derivatives. Free Radic Res 2006;40:11051111.

31 Ebrahimi A, Schluesener H: Natural polyphenols against neurodegenerative disorders: potentials and pitfalls. Ageing Res Rev 2012;11:329-345. 